\title{
Existence for Nonautonomous Fractional Integrodifferential Equations with Nonlocal Conditions
}

\author{
Fang Li \\ School of Mathematics, Yunnan Normal University, Kunming 650092, China \\ Correspondence should be addressed to Fang Li; fangli860@gmail.com
}

Received 10 July 2013; Revised 16 September 2013; Accepted 12 October 2013

Academic Editor: Dumitru Baleanu

Copyright (C) 2013 Fang Li. This is an open access article distributed under the Creative Commons Attribution License, which permits unrestricted use, distribution, and reproduction in any medium, provided the original work is properly cited.

\begin{abstract}
We study the existence of mild solution of a class of nonlinear nonautonomous fractional integrodifferential equations with nonlocal conditions in a separable Banach space $X$. Combining the techniques of operator semigroup, noncompactness measures, and the fixed point theory, we obtain new existence of mild solution without the assumptions that the nonlinearity $f$ satisfies a Lipschitz type condition and the semigroup $\{\exp (-t A(s))\}$ generated by $\{-A(s)\}_{s \in[0, T]}$ is compact. An application of the abstract result is also given.
\end{abstract}

\section{Introduction}

In this paper, we denote that $C$ is a positive constant and assume that a family of closed linear operators $\{A(t)\}_{t \in[0, T]}$ satisfies the following.

(A1) The domain $D(A)$ of $\{A(t)\}_{t \in[0, T]}$ is dense in a Banach space $X$ and independent of $t$.

(A2) The operator $[A(t)+\lambda]^{-1}$ exists in $L(X)$ (the Banach space of all linear and bounded operators on $X)$ for any $\lambda$ with $\operatorname{Re} \lambda \geq 0$ and

$$
\left\|[A(t)+\lambda]^{-1}\right\| \leq \frac{C}{|\lambda|+1}, \quad t \in[0, T] .
$$

(A3) There exist constants $\gamma \in(0,1]$ and $C$ such that

$$
\begin{array}{r}
\left\|\left[A\left(t_{1}\right)-A\left(t_{2}\right)\right] A^{-1}(s)\right\| \leq C\left|t_{1}-t_{2}\right|^{\gamma}, \\
t_{1}, t_{2}, s \in[0, T] .
\end{array}
$$

Under condition (A2), each operator $-A(s), s \in[0, T]$, generates an analytic semigroup $\exp (-t A(s)), t>0$, and there exists a constant $C$ such that

$$
\left\|A^{n}(s) \exp (-t A(s))\right\| \leq \frac{C}{t^{n}},
$$

where $n=0,1, t>0, s \in[0, T]([1])$.
Moreover, $\left\|A(t) A^{-1}(s)\right\| \leq C$, which follows from condition (A2), where $t, s \in[0, T]$ and $C$ is a positive constant independent of both $t$ and $s$.

This paper is concerned with existence result for nonautonomous fractional integrodifferential equations with nonlocal conditions in a separable Banach space $X$ :

$$
\begin{gathered}
\frac{d^{q} v(t)}{d t^{q}}=-A(t) v(t)+f\left(t, \int_{0}^{t} K(t, s) v(s) d s, v(t)\right), \\
t \in[0, T], \\
v(0)=A^{-1}(0) g(v),
\end{gathered}
$$

where $T>0,0<q<1,\{A(t)\}_{t \in[0, T]}$ is a family of closed linear operators in $X$ and satisfies (A1)-(A3), $K \in C\left(\Delta, \mathbf{R}^{+}\right)$with $\Delta=\left\{(t, s) \in \mathbf{R}^{2}: 0 \leq s \leq t \leq T\right\}$ and

$$
\sup _{t \in[0, T]} \int_{0}^{t} K(t, s) d s<\infty,
$$

$f:[0, T] \times X \times X \rightarrow X, g$ are given functions to be specified later. The fractional derivative is understood here in the Riemann-Liouville sense.

Fractional calculus is a generalization of ordinary differentiation and integration to arbitrary noninteger order. The field of the application of fractional calculus is very broad. 
We can see it in the study of the memorial materials, earthquake analysis, robots, electric fractal network, fractional sine oscillator, electrolysis chemical, fractional capacitance theory, electrode electrolyte interface description, fractal theory, especially in the dynamic process description of porous structure, fractional controller design, vibration control of viscoelastic system and pliable structure objects, fractional biological neurons, and probability theory. For details, see the monographs of Kilbas et al. [2], Kiryakova [3], Lakshmikantham and Vatsala [4], Miller and Ross [5], Samko et al. [6] and Podlubny [7], and the references therein. Some recent contributions to the theory of fractional differential equations can be seen in [8-20] and the references therein. Among the previous researches, most of researchers focus on the case that the differential operators (possibly unbounded) in the main parts are independent of time $t$. However, when treating some parabolic evolution problems, it is usually assumed that the partial differential operators depend on time $t$ (i.e., it is the case of the problems under considerations being nonautonomous), since this class of operators appears frequently in the applications (see [21] and the references therein).

Moreover, since the work of Byszewski [22], the nonlocal Cauchy problems have been investigated in many papers (cf., e.g., $[13-15,20,23-25]$ and the references therein). The nonlocal conditions give a better description in applications than standard ones, and the Cauchy problem with nonlocal initial condition can be applied in physics with better effect than the classical Cauchy problem with traditional initial conditions. The existence of mild solutions of nonautonomous fractional evolution equations with nonlocal conditions of the form (4) is an untreated original topic, which in fact is the main motivation of the present paper.

In this paper, using a pair of evolution families $\{\psi(t, s)\}$ and $\{\varphi(t, s)\}$ associated with the semigroup $\{\exp (-t A(s))\}(t>$ 0 ), we give a reasonable concept of solution to problem (4) in Section 2. Moreover, in general, the semigroup $\{\exp (-t A(s))\}$ $(t>0)$ generated by $-A(s)(s \in[0, T])$ is not compact, so we obtain the main result based on the theory of measures of noncompactness and the condensing maps. These techniques are often used to deal with abstract integer order differential equations but rarely used in abstract fractional order differential equations(e.g., [8-20] and the references therein). We will study (4) under suitable hypotheses based on a special noncompactness measure and the properties of fixed points set of condensing operators $[26,27]$ and establish a new existence result for (4) without the assumptions that the nonlinearity $f$ satisfies a Lipschitz type condition and the semigroup $\{\exp (-t A(s))\}$ generated by $-A(s)$ is compact (see Theorem 14). As one can see, our result is obtained under assumptions weaker than those required previously in the similar literature. The result is new even for the case of $A(t) \equiv$ $A$ (autonomous). Moreover, an example is given to show an application of the abstract result.

\section{Preliminaries}

Throughout this paper, we set $J=[0, T]$, a compact interval in R. We denote by $X$ a separable Banach space with norm $\|\cdot\|$, by $L(X)$ the Banach space of all linear and bounded operators on $X$, and by $C([a, b], X)$ the space of all $X$-valued continuous functions on $[a, b]$ with the supremum norm as follows:

$$
\begin{array}{r}
\|x\|_{[a, b]}=\|x\|_{C([a, b], X)}=\sup \{\|x(t)\|: t \in[a, b]\}, \\
\text { for any } x \in C([a, b], X) .
\end{array}
$$

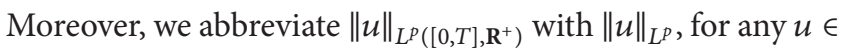
$L^{p}\left([0, T], \mathbf{R}^{+}\right)$.

We set

$$
\begin{gathered}
G v(t):=\int_{0}^{t} K(t, s) v(s) d s, \\
G^{*}:=\sup _{t \in J} \int_{0}^{t} K(t, s) d s<\infty .
\end{gathered}
$$

Next, we recall the definition of the Riemann-Liouville integral.

Definition 1 (see [6]). The fractional (arbitrary) order integral of the function $F \in L^{1}\left(\mathbf{R}^{+}, \mathbf{R}\right)$ of order $\alpha>0$ is defined by

$$
I^{\alpha} F(t)=\frac{1}{\Gamma(\alpha)} \int_{0}^{t}(t-s)^{\alpha-1} F(s) d s,
$$

where $\Gamma$ is the Gamma function. Moreover, $I^{\alpha_{1}} I^{\alpha_{2}}=I^{\alpha_{1}+\alpha_{2}}$, for all $\alpha_{1}, \alpha_{2}>0$.

Remark 2. We have (1) $I^{\alpha}: L^{1}[0, T] \rightarrow L^{1}[0, T][6]$.

(2) Obviously, for $F \in L^{1}(J, \mathbf{R})$, it follows from Definition 1 that

$$
\begin{aligned}
\int_{0}^{t} \int_{0}^{\eta}(t-\eta)^{q-1}(\eta-s)^{\gamma-1} F(s) d s d \eta \\
\quad=B(q, \gamma) \int_{0}^{t}(t-s)^{q+\gamma-1} F(s) d s,
\end{aligned}
$$

where $B(q, \gamma)$ is a beta function.

Definition 3 (see [7]). The Riemann-Liouville derivative of order $q$ with the lower limit zero for a function $F \in A C[0, \infty)$ can be written as

$$
\begin{array}{r}
{ }^{L} D_{t}^{q} F(t)=\frac{1}{\Gamma(1-q)} \frac{d}{d t} \int_{0}^{t}(t-s)^{-q} F(s) d s, \\
t>0,0<q<1 .
\end{array}
$$

Based on the work in [12], we give the following definition of the operator family $\{\psi(t, s)\}$.

Definition 4. Let $\xi_{q}$ be a probability density function defined on $(0, \infty)$ such that its Laplace transform is given by

$$
\int_{0}^{\infty} e^{-\sigma x} \xi_{q}(\sigma) d \sigma=\sum_{j=0}^{\infty} \frac{(-x)^{j}}{\Gamma(1+q j)}, \quad 0<q \leq 1, x>0 .
$$

We define operator families $\{\psi(t, s)\}$ by the semigroup $\exp (-t A(s))$ associated with $A(s)$ as follows:

$$
\psi(t, s)=q \int_{0}^{\infty} \theta t^{q-1} \xi_{q}(\theta) \exp \left(-t^{q} \theta A(s)\right) d \theta .
$$


By using the family $\{\psi(t, s)\}$, we denote

$$
\begin{gathered}
\varphi_{1}(t, s)=[A(t)-A(s)] \psi(t-s, s), \\
\varphi_{k+1}(t, s)=\int_{s}^{t} \varphi_{k}(t, \theta) \varphi_{1}(\theta, s) d \theta, \quad k=1,2, \ldots,
\end{gathered}
$$

and construct the family $\{\varphi(t, s)\}$ by

$$
\varphi(t, s)=\sum_{k=1}^{\infty} \varphi_{k}(t, s) .
$$

Lemma 5 (see [12]). The operator-valued functions $\psi(t-s, s)$ and $A(t) \psi(t-s, s)$ are continuous in uniform topology in the variables $t, s$, where $0 \leq s \leq t-\varepsilon, 0 \leq t \leq T$, for any $\varepsilon>0$. Clearly,

$$
\|\psi(t-s, s)\| \leq C(t-s)^{q-1} .
$$

Moreover, we have

$$
\|\varphi(t, s)\| \leq C(t-s)^{\gamma-1}
$$

A mild solution of (4) can be defined as follows.

Definition 6. A function $v \in C(J, X)$ satisfying the equation

$$
\begin{aligned}
v(t)= & A^{-1}(0) g(v)+\int_{0}^{t} \psi(t-s, s) f(s, G v(s), v(s)) d s \\
& +\int_{0}^{t} \psi(t-s, s) U(s) g(v) d s \\
& +\int_{0}^{t} \int_{0}^{\eta} \psi(t-\eta, \eta) \varphi(\eta, s) f(s, G v(s), v(s)) d s d \eta
\end{aligned}
$$

is called a mild solution of (4), where

$$
\begin{gathered}
U(t)=-A(t) A^{-1}(0)-\int_{0}^{t} \varphi(t, s) A(s) A^{-1}(0) d s, \\
\|U(t)\| \leq C+C t^{\gamma} .
\end{gathered}
$$

We will need the following facts from the theory of measures of noncompactness and condensing maps (see, e.g., [26, 27]) which are used later in this paper.

Definition 7. Let $E$ be a Banach space, $2^{E}$ the family of all nonempty subsets of $E,(\mathscr{A}, \geq)$ a partially ordered set, and $v: 2^{E} \rightarrow \mathscr{A}$. If, for every $\Omega \in 2^{E}$,

$$
\nu(\overline{\mathrm{CO}}(\Omega))=\nu(\Omega),
$$

then we say that $v$ is a measure of noncompactness (MNC) in E.

As an example of the MNC, we may consider the Hausdorff MNC:

$$
\chi(\Omega)=\inf \{\varepsilon>0: \Omega \text { has a finite } \varepsilon \text {-net }\} .
$$

We know that $\chi$ is monotone, nonsingular, invariant with respect to union with compact sets, algebraically semiadditive, and regular. This means that

(i) for any $\Omega_{0}, \Omega_{1} \in 2^{E}$ with $\Omega_{0} \subset \Omega_{1}, \chi\left(\Omega_{0}\right) \leq \chi\left(\Omega_{1}\right)$,

(ii) for any $a_{0} \in E, \Omega \in 2^{E}, \chi\left(\left\{a_{0}\right\} \cup \Omega\right)=\chi(\Omega)$, (iii) for every relatively compact set $D \subset E, \Omega \in 2^{E}$, $\chi(\{D\} \cup \Omega)=\chi(\Omega)$,

(iv) for each $\Omega_{0}, \Omega_{1} \in 2^{E}, \chi\left(\Omega_{0}+\Omega_{1}\right) \leq \chi\left(\Omega_{0}\right)+\chi\left(\Omega_{1}\right)$,

(v) $v(\Omega)=0$ is equivalent to the relative compactness of $\Omega$.

In Section 3, we will establish an existence result to the problem (4) using the following assertion about $\chi$-estimates for a multivalued integral (Theorem 4.2.3 of [27]).

Let $\mathscr{G}:[0, \tilde{h}] \rightarrow 2^{E}$ be a multifunction. It is called

(i) integrable, if it admits a Bochner integrable selection $\mathfrak{g}:[0, \tilde{h}] \rightarrow E, \mathfrak{g}(t) \in \mathscr{G}(t)$ for a.e. $t \in[0, \tilde{h}] ;$

(ii) integrably bounded, if there exists a function $\varrho \in$ $L^{1}([0, \tilde{h}], E)$ such that

$$
\|\mathscr{G}(t)\|:=\sup \{\|\mathfrak{g}\|: \mathfrak{g} \in \mathscr{G}(t)\} \leq \varrho(t) \quad \text { a.e. } t \in[0, \tilde{h}] .
$$

Proposition 8. For an integrable, integrably bounded multifunction $\mathscr{G}:[0, \tilde{h}] \rightarrow 2^{X}$ where $X$ is a separable Banach space, let

$$
\chi(\mathscr{G}(t)) \leq \mathfrak{q}(t), \quad \text { for a.e. } t \in[0, \widetilde{h}],
$$

where $\mathfrak{q} \in L_{+}^{1}([0, \tilde{h}])$. Then $\chi\left(\int_{0}^{t} \mathscr{G}(s) d s\right) \leq \int_{0}^{t} \mathfrak{q}(s) d s$ for all $t \in[0, \tilde{h}]$.

Let $E$ be a Banach space and $v$ a monotone nonsingular MNC in $E$.

Definition 9. A continuous map $\mathfrak{B}: Y \subseteq E \rightarrow E$ is called condensing with respect to a MNC $\nu$ (or $\nu$-condensing) if, for every bounded set $\Omega \subseteq Y$ which is not relatively compact, we have

$$
\nu(\mathfrak{B}(\Omega)) \nsupseteq \nu(\Omega) .
$$

The application of the topological degree theory for condensing maps (see, e.g., [26, 27]) yields the following fixed point principle which will be used later.

Theorem 10. Let $\mathfrak{M}$ be a bounded convex closed subset of $E$ and $\mathfrak{B}: \mathfrak{M} \rightarrow \mathfrak{M}$ a $v$-condensing map. Then fix $\mathfrak{B}=\{x: x=$ $\mathfrak{B}(x)\}$ is nonempty.

\section{Main Result}

We need the hypotheses as follows.

(H1) Function $f: J \times X \times X \rightarrow X$ satisfies that $f(\cdot, u, w)$ : $J \rightarrow X$ is measurable for all $(u, w) \in X \times X$ and $f(t, \cdot, \cdot): X \times X \rightarrow X$ is continuous for a.e. $t \in J$, and there exists a function $\mu(\cdot) \in L^{\bar{p}}\left(J, \mathbf{R}^{+}\right)(\bar{p}>1 / q>1)$ such that

$$
\begin{array}{r}
\|f(t, u, w)\| \leq \mu(t)(\|u\|+\|w\|), \\
(t, v, w) \in J \times X \times X,
\end{array}
$$

for almost all $t \in J$. 
(H2) There exists a function $\delta \in L^{p}\left(J, \mathbf{R}^{+}\right)$such that, for any bounded set $D \subset X$,

$$
\chi(f(t, G D, D)) \leq \delta(t) \chi(D), \quad \text { a.e. } t \in[0, T],
$$

where $p>\max \{1 / q, 1 / \gamma\}>1$.

(H3) The function $g$ is completely continuous and there exists a positive constant $b$ such that

$$
\|g(v)\| \leq b, \quad v \in C(J, X) .
$$

Define the operator $\mathscr{F}: C(J, X) \rightarrow C(J, X)$ as follows:

$$
\begin{aligned}
(\mathscr{F} v)(t)= & A^{-1}(0) g(v)+\int_{0}^{t} \psi(t-s, s) U(s) g(v) d s \\
& +\int_{0}^{t} \psi(t-s, s) f(s, G v(s), v(s)) d s \\
& +\int_{0}^{t} \int_{0}^{\sigma} \psi(t-\sigma, \sigma) \varphi(\sigma, s) \\
& \times f(s, G v(s), v(s)) d s d \sigma .
\end{aligned}
$$

It is clear that the operator $\mathscr{F}$ is well defined. For some MNC $\nu$, we will show that the operator $\mathscr{F}$ is $\nu$-condensing on every bounded subset of $C(J, X)$. To this end, we divided the proof into three propositions.

Proposition 11. The operator $\mathscr{F}$ is continuous.

Proof. Let $\left\{v_{n}\right\}_{n \in \mathbf{N}}$ be a sequence such that $v_{n} \rightarrow v$ in $C(J, X)$ as $n \rightarrow \infty$. Since $f$ satisfies (H1), for almost every $t \in J$ and $(t, s) \in \Delta$, we have

$$
f\left(t, G v_{n}(t), v_{n}(t)\right) \longrightarrow f(t, G v(t), v(t)), \quad \text { as } n \longrightarrow \infty .
$$

For $t \in J$, we can prove that $\mathscr{F}$ is continuous. In fact, noting (H1) and $v_{n} \rightarrow v$ in $C(J, X)$, we know that there exists $\varepsilon>0$ such that $\left\|v_{n}-v\right\|_{J} \leq \varepsilon$ for $n$ sufficiently large. Therefore, we get

$$
\begin{aligned}
& \left\|f\left(t, G v_{n}(t), v_{n}(t)\right)-f(t, G v(t), v(t))\right\| \\
& \quad \leq \mu(t)\left(G^{*}+1\right)\left[\left\|v_{n}-v\right\|_{J}+2\|v\|_{J}\right] \\
& \quad \leq \mu(t)\left(G^{*}+1\right)\left[\varepsilon+2\|v\|_{J}\right] .
\end{aligned}
$$

In view of (15) and (16), we obtain

$$
\begin{array}{r}
\int_{0}^{t} \| \psi(t-s, s)\left[f\left(s, G v_{n}(s), v_{n}(s)\right)\right. \\
-f(s, G v(s), v(s))] \| d s \\
+\int_{0}^{t} \int_{0}^{\sigma} \| \psi(t-\sigma, \sigma) \varphi(\sigma, s) \\
\times\left[f\left(s, G v_{n}(s), v_{n}(s)\right)\right. \\
-f(s, G v(s), v(s))] \| d s
\end{array}
$$

$$
\begin{aligned}
& \leq C \int_{0}^{t}(t-s)^{q-1} \\
& \quad \times \| f\left(s, G v_{n}(s), v_{n}(s)\right) \\
& \quad-f(s, G v(s), v(s)) \| d s \\
& +C^{2} \int_{0}^{t} \int_{0}^{\sigma}(t-\sigma)^{q-1}(\sigma-s)^{\gamma-1} \\
& \quad \times \| f\left(s, G v_{n}(s), v_{n}(s)\right) \\
& \quad-f(s, G v(s), v(s)) \| d s
\end{aligned}
$$$$
\longrightarrow 0, \quad \text { as } n \longrightarrow \infty \text {. }
$$

Therefore, the fact $\left\|A^{-1}(0)\right\| \leq C$ (by (A2)), (H3), and the Lebesgue dominated convergence theorem ensure that

$$
\left\|\left(\mathscr{F} v_{n}\right)(t)-(\mathscr{F} v)(t)\right\| \longrightarrow 0, \quad \text { as } n \longrightarrow \infty .
$$

Therefore, we deduce that

$$
\lim _{n \rightarrow \infty}\left\|\mathscr{F} v_{n}-\mathscr{F} v\right\|_{J}=0 .
$$

Proposition 12. The operator $\mathscr{F}$ transforms bounded sets into equicontinuous ones.

Proof. For any $r>0$, we set $B_{r}=\left\{v \in X:\|v\|_{J} \leq r\right\}$. Let $0<t_{2}<t_{1}<T$ and $v \in B_{r}$. Then

$$
\left\|(\mathscr{F} v)\left(t_{1}\right)-(\mathscr{F} v)\left(t_{2}\right)\right\| \leq \sum_{i=1}^{6} I_{i}
$$

where

$$
\begin{gathered}
I_{1}=\int_{0}^{t_{2}}\left\|\left[\psi\left(t_{1}-s, s\right)-\psi\left(t_{2}-s, s\right)\right] U(s) g(v)\right\| d s, \\
I_{2}=\int_{t_{2}}^{t_{1}}\left\|\psi\left(t_{1}-s, s\right) U(s) g(v)\right\| d s, \\
I_{3}=\int_{0}^{t_{2}} \|\left[\psi\left(t_{1}-s, s\right)-\psi\left(t_{2}-s, s\right)\right] \\
\quad \times f(s, G v(s), v(s)) \| d s,
\end{gathered}
$$

$$
\begin{gathered}
I_{4}=\int_{t_{2}}^{t_{1}}\left\|\psi\left(t_{1}-s, s\right) f(s, G v(s), v(s))\right\| d s, \\
I_{5}=\int_{0}^{t_{2}} \int_{0}^{\sigma} \|\left[\psi\left(t_{1}-\sigma, \sigma\right)-\psi\left(t_{2}-\sigma, \sigma\right)\right] \\
\quad \times \varphi(\sigma, s) f(s, G v(s), v(s)) \| d s d \sigma, \\
I_{6}=\int_{t_{2}}^{t_{1}} \int_{0}^{\sigma}\left\|\psi\left(t_{1}-\sigma, \sigma\right) \varphi(\sigma, s) f(s, G v(s), v(s))\right\| d s d \sigma .
\end{gathered}
$$


For $I_{1}$,

$$
\begin{aligned}
I_{1}= & \left(\int_{0}^{t_{2}-\varepsilon}+\int_{t_{2}-\varepsilon}^{t_{2}}\right) \\
& \times\left\|\left[\psi\left(t_{1}-s, s\right)-\psi\left(t_{2}-s, s\right)\right] U(s) g(v)\right\| d s \\
= & I_{1}^{\prime}+I_{1}^{\prime \prime} .
\end{aligned}
$$

It follows from Lemma 5, (19), and (H3) that $I_{1}^{\prime} \rightarrow 0$ as $t_{2} \rightarrow$ $t_{1}$. For $I_{1}^{\prime \prime}$, from (15) and (19),

$$
\begin{aligned}
I_{1}^{\prime \prime} & \leq C^{2} b\left\{\int_{t_{2}-\varepsilon}^{t_{2}}\left[\left(t_{1}-s\right)^{q-1}+\left(t_{2}-s\right)^{q-1}\right]\left(1+s^{\gamma}\right) d s\right\} \\
& \longrightarrow 0, \quad \text { as } t_{2} \longrightarrow t_{1}, \varepsilon \longrightarrow 0 .
\end{aligned}
$$

Similarly, $I_{3}, I_{5} \rightarrow 0$, as $t_{2} \rightarrow t_{1}$ and $\varepsilon \rightarrow 0$. For $I_{2}$, from (15) and (19), we have

$$
I_{2} \leq b \int_{t_{2}}^{t_{1}} C^{2}\left(t_{1}-s\right)^{q-1}\left(1+s^{\gamma}\right) d s \longrightarrow 0, \quad \text { as } t_{2} \longrightarrow t_{1} .
$$

Similarly, $I_{4}, I_{6} \rightarrow 0$, as $t_{2} \rightarrow t_{1}$.

So, the set $\left\{(\mathscr{F} v)(\cdot): v \in B_{r}\right\}$ is equicontinuous.

Proposition 13. The operator $\mathscr{F}$ is $\nu$-condensing.

Proof. Noting that, for any $\phi \in L^{1}(J, X)$, we have

$$
\lim _{L \rightarrow+\infty} \sup _{t \in J} \int_{0}^{t} e^{-L(t-s)} \phi(s) d s=0,
$$

so, we can take the appropriate $L$ such that

$$
\begin{gathered}
C^{2}\left(\frac{p-1}{p \gamma-1}\right)^{(p-1) / p} \\
\quad \times\|\delta\|_{L^{p}} \sup _{t \in J} \int_{0}^{t}(t-\sigma)^{q-1} \sigma^{\gamma-1 / p} e^{-L(t-\sigma)} d \sigma \\
=L_{1}<\frac{1}{2}, \\
\quad C \sup _{t \in J} \int_{0}^{t}(t-s)^{q-1} \delta(s) e^{-L(t-s)} d s=L_{2}<\frac{1}{2}, \\
C\left(G^{*}+1\right) \sup _{t \in J} \int_{0}^{t}(t-s)^{q-1} \mu(s) \\
\quad \times\left(1+C B(q, \gamma)(t-s)^{\gamma}\right) e^{-L(t-s)} d s \\
=L_{3}<1 .
\end{gathered}
$$

For every bounded subset $\Omega \subset C(J, X)$, we consider the measure of noncompactness $v$ in the space $C(J, X)$ with values in the cone $\mathbf{R}_{+}^{2}$ of the following way:

$$
\nu(\Omega)=\left(\Psi(\Omega), \bmod _{c}(\Omega)\right),
$$

where $\bmod _{c}(\Omega)$ is the module of equicontinuity of $\Omega$ given by

$$
\begin{aligned}
\bmod _{c}(\Omega) & =\lim _{\epsilon \rightarrow 0} \sup _{v \in \Omega} \max _{\left|t_{1}-t_{2}\right| \leq \epsilon}\left\|v\left(t_{1}\right)-v\left(t_{2}\right)\right\|, \\
\Psi(\Omega) & =\sup _{t \in J}\left(e^{-L t} \chi(\Omega(t))\right) .
\end{aligned}
$$

Let $\Omega \subset C(J, X)$ be a nonempty, bounded set such that

$$
\nu(\mathscr{F}(\Omega)) \geq \nu(\Omega) .
$$

For any $t \in[0, \eta]$, we set

$$
\widehat{\mathscr{F}}_{1}(\Omega)(t)=\left\{\int_{0}^{t} \varphi(t, s) f(s, G v(s), v(s)) d s: v \in \Omega\right\} .
$$

We consider the multifunction $s \in[0, t] \multimap H(s)$,

$$
H(s)=\{\varphi(t, s) f(s, G v(s), v(s)): v \in \Omega\} .
$$

Obviously, $H$ is integrable, and from (16) and (H1) it follows that $H$ is integrably bounded. Moreover, noting $(\mathrm{H} 2)$, we have the following estimate for a.e. $s \in[0, t]$ :

$$
\begin{aligned}
\chi(H(s)) & \leq C(t-s)^{\gamma-1} \chi(f(s, G \Omega(s), \Omega(s))) \\
& \leq C(t-s)^{\gamma-1} \delta(s) \chi(\Omega(s)) \\
& =C(t-s)^{\gamma-1} \delta(s) e^{L s} e^{-L s} \chi(\Omega(s)) \\
& \leq C(t-s)^{\gamma-1} \delta(s) e^{L s} \Psi(\Omega) .
\end{aligned}
$$

Applying Proposition 8, we have

$$
\begin{aligned}
\chi\left(\widehat{\mathscr{F}}_{1}(\Omega)(t)\right) & =\chi\left(\int_{0}^{t} H(s) d s\right) \\
& \leq C \int_{0}^{t}(t-s)^{\gamma-1} \delta(s) e^{L s} d s \cdot \Psi(\Omega) .
\end{aligned}
$$

Set

$$
\begin{aligned}
\widehat{\mathscr{F}}_{2}(\Omega)(\sigma)=\{ & \psi(t-\sigma, \sigma) \\
& \left.\times \int_{0}^{\sigma} \varphi(\sigma, s) f(s, G v(s), v(s)) d s: v \in \Omega\right\} .
\end{aligned}
$$

Then from (49),

$$
\begin{aligned}
& \chi\left(\widehat{\mathscr{F}}_{2}(\Omega)(\sigma)\right) \\
& \quad \leq C^{2}(t-\sigma)^{q-1} \int_{0}^{\sigma}(\sigma-s)^{\gamma-1} \delta(s) e^{L s} d s \cdot \Psi(\Omega),
\end{aligned}
$$


Furthermore

$$
\begin{aligned}
\chi\left(\int_{0}^{t} \widehat{\mathscr{F}}_{2}(\Omega)(\sigma) d \sigma\right) \\
\leq C^{2} \int_{0}^{t}(t-\sigma)^{q-1} \int_{0}^{\sigma}(\sigma-s)^{\gamma-1} \delta(s) e^{L s} d s d \sigma \cdot \Psi(\Omega) \\
\leq C^{2} \int_{0}^{t}(t-\sigma)^{q-1} e^{L \sigma}\left(\int_{0}^{\sigma}(\sigma-s)^{\gamma-1} \delta(s) d s\right) d \sigma \cdot \Psi(\Omega) \\
\leq C^{2}\left(\frac{p-1}{p \gamma-1}\right)^{(p-1) / p} \\
\quad \times\|\delta\|_{L^{p}} \int_{0}^{t}(t-\sigma)^{q-1} \sigma^{\gamma-1 / p} e^{L \sigma} d \sigma \cdot \Psi(\Omega) .
\end{aligned}
$$

Therefore, combining with (40), we have

$$
\begin{aligned}
\sup _{t \in J}\left(e^{-L t} \chi\left(\int_{0}^{t} \widehat{\mathscr{F}}_{2}(\Omega)(\sigma) d \sigma\right)\right) \\
\leq C^{2}\left(\frac{p-1}{p \gamma-1}\right)^{(p-1) / p}\|\delta\|_{L^{p}} \\
\quad \times \sup _{t \in J} \int_{0}^{t}(t-\sigma)^{q-1} \sigma^{\gamma-1 / p} e^{-L(t-\sigma)} d \sigma \cdot \Psi(\Omega) \\
=L_{1} \Psi(\Omega) .
\end{aligned}
$$

For any $t \in J$, we set

$$
\widehat{\mathscr{F}}_{3}(\Omega)(t)=\left\{\int_{0}^{t} \psi(t-s, s) f(s, G v(s), v(s)) d s: v \in \Omega\right\}
$$

and consider the multifunction $s \in[0, t] \multimap \widetilde{H}(s)$ :

$$
\widetilde{H}(s)=\{\psi(t-s, s) f(s, G v(s), v(s)): v \in \Omega\} .
$$

Obviously, $\widetilde{H}$ is integrable, and from (15) and (H1) it follows that $\widetilde{H}$ is integrably bounded. Moreover, for a.e. $s \in[0, t]$, we can obtain

$$
\begin{aligned}
\chi(\widetilde{H}(s)) & \leq C(t-s)^{q-1} \delta(s) e^{L s} \Psi(\Omega) \\
\chi\left(\widehat{\mathscr{F}}_{3}(\Omega)(t)\right) & =\chi\left(\int_{0}^{t} \widetilde{H}(s) d s\right) \\
& \leq C \int_{0}^{t}(t-s)^{q-1} \delta(s) e^{L s} d s \cdot \Psi(\Omega)
\end{aligned}
$$

by the similar technique used in (49)-(53); then

$$
\begin{aligned}
\sup _{t \in J}\left(e^{-L t} \chi\left(\widehat{\mathscr{F}}_{3}(\Omega)(t)\right)\right) \\
\quad \leq C \sup _{t \in J} \int_{0}^{t}(t-s)^{q-1} \delta(s) e^{-L(t-s)} d s \cdot \Psi(\Omega) \\
\quad=L_{2} \Psi(\Omega) .
\end{aligned}
$$

Now, from (53) and (57), $L>0$ can be chosen so that

$$
\Psi(\mathscr{F}(\Omega)) \leq\left(L_{1}+L_{2}\right) \Psi(\Omega)=\widetilde{L} \Psi(\Omega)
$$

where $0<\widetilde{L}<1$. Then from (45), we have $\Psi(\Omega)=0$.

Further, from Proposition 12 we know that $\bmod _{c}(\mathscr{F}(\Omega))=0$ and integrating with (45) one yields $\bmod _{c}(\Omega)=0$. Hence $v(\Omega)=(0,0)$. The regularity property of $\nu$ implies the relative compactness of $\Omega$. Now, it follows from Definition 9 that $\mathscr{F}$ is $\nu$-condensing.

Theorem 14. Assume that (H1), (H2), and (H3) are satisfied; then problem (4) has at least one mild solution on the interval $[0, T]$.

Proof. Let us introduce in the space $C(J, X)$ the equivalent norm defined as

$$
\|v\|_{*}=\sup _{t \in J}\left(e^{-L t}\|v(t)\|\right)
$$

Consider the set

$$
B_{r}=\left\{v \in C(J, X):\|v\|_{*} \leq r\right\} .
$$

Next, we show that there exists some $r>0$ such that $\mathscr{F} B_{r} \subset B_{r}$. Suppose on the contrary that for each $r>0$ there exist $v_{r}(\cdot) \in B_{r}$ and some $t \in J$ such that $\left\|\left(\mathscr{F} v_{r}\right)(t)\right\|_{*}>r$.

Combining with (H1)-(H3), Remark 2(2), and (15), (16) and (19), we have

$$
\begin{aligned}
& \left\|\left(\mathscr{F} v_{r}\right)(t)\right\| \\
& \leq\left\|A^{-1}(0) g\left(v_{r}\right)\right\| \\
& +\int_{0}^{t}\left\|\psi(t-s, s) U(s) g\left(v_{r}\right)\right\| d s \\
& +\int_{0}^{t}\left\|\psi(t-s, s) f\left(s, G v_{r}(s), v_{r}(s)\right)\right\| d s \\
& +\int_{0}^{t} \int_{0}^{\sigma} \| \psi(t-\sigma, \sigma) \varphi(\sigma, s) \\
& \times f\left(s, G v_{r}(s), v_{r}(s)\right) \| d s d \sigma \\
& \leq C b+C^{2} b \int_{0}^{t}(t-s)^{q-1}\left(1+s^{\gamma}\right) d s \\
& +C G^{*} \int_{0}^{t}(t-s)^{q-1} \mu(s) e^{L s} d s \cdot\left\|v_{r}\right\|_{*} \\
& +C \int_{0}^{t}(t-s)^{q-1} \mu(s)\left\|v_{r}(s)\right\| d s \\
& +C^{2} G^{*} B(q, \gamma) \int_{0}^{t}(t-s)^{q+\gamma-1} \mu(s) e^{L s} d s \cdot\left\|v_{r}\right\|_{*} \\
& +C^{2} B(q, \gamma) \int_{0}^{t}(t-s)^{q+\gamma-1} \mu(s)\left\|v_{r}(s)\right\| d s
\end{aligned}
$$




$$
\begin{aligned}
\leq & C b+C^{2} b \int_{0}^{t}(t-s)^{q-1}\left(1+s^{\gamma}\right) d s \\
& +C\left(G^{*}+1\right) \cdot r \int_{0}^{t}(t-s)^{q-1} \mu(s) e^{L s} d s \\
& +C^{2}\left(G^{*}+1\right) \cdot r B(q, \gamma) \int_{0}^{t}(t-s)^{q+\gamma-1} \mu(s) e^{L s} d s .
\end{aligned}
$$

Therefore

$$
\begin{aligned}
r< & \sup _{t \in J}\left(e^{-L t}\left\|\left(\mathscr{F} v_{r}\right)(t)\right\|\right) \\
\leq & \sup _{t \in J} M(t)+C\left(G^{*}+1\right) \\
& \cdot r \sup _{t \in J} \int_{0}^{t}(t-s)^{q-1} \mu(s) e^{-L(t-s)} d s \\
& +C^{2}\left(G^{*}+1\right) \cdot r B(q, \gamma) \\
& \times \sup _{t \in J} \int_{0}^{t}(t-s)^{q+\gamma-1} \mu(s) e^{-L(t-s)} d s,
\end{aligned}
$$

where $M(t)=C b\left(1+C \int_{0}^{t}(t-s)^{q-1}\left(1+s^{\gamma}\right) d s\right)$.

Dividing both sides of (62) by $r$ and taking $r \rightarrow \infty$, we have

$$
\begin{aligned}
C\left(G^{*}+1\right) \sup _{t \in J} \int_{0}^{t}(t-s)^{q-1} \mu(s) \\
\quad \times\left(1+C B(q, \gamma)(t-s)^{\gamma}\right) e^{-L(t-s)} d s \geq 1 .
\end{aligned}
$$

This contradicts (42). Hence for some positive number $r$, $\mathscr{F} B_{r} \subset B_{r}$. From Proposition 13 it follows that $\mathscr{F}$ is $\nu$-condensing and we apply Theorem 10 to complete the proof.

\section{Example}

In this section, set $X=L^{2}([0,1], \mathbf{R})$, and we consider the following integrodifferential problem:

$$
\begin{aligned}
\frac{\partial^{q}}{\partial t^{q}} u(t, \xi)= & a(t, \xi) \frac{\partial^{2} u}{\partial \xi^{2}}(t, \xi) \\
& +\sin \left(\int_{0}^{t}(t-s) u(s, \xi) d s\right) \\
& +\frac{1}{k \sqrt[k]{t}} \cdot \frac{u(t, \xi)}{1+u(t, \xi)}, \\
u(t, 0)=u(t, 1)=0 & (a(0, \xi))^{-1} \\
u(0, \xi)=\left(\sum^{j} \int_{0}^{1} c_{i}(\xi, y) \frac{u\left(t_{i}, y\right)}{1+u\left(t_{i}, y\right)} d y\right. &
\end{aligned}
$$

where $\partial^{q} / \partial t^{q}$ is the Riemann-Liouville fractional partial derivative of order $0<q<1, t \in[0,1], \xi \in[0,1], k>0$ is a constant to be specified later. Also $0<t_{0}<t_{1}<\cdots<t_{j}<$ $1\left(j \in \mathbf{N}^{+}\right), c_{i}(\cdot, \cdot)(i=0,1, \ldots, j)$ are continuous functions and there exists a positive constant $b$ such that

$$
\sum_{i=0}^{j} \int_{0}^{1}\left\|c_{i}(\xi, y)\right\| d y \leq b .
$$

$a(t, \xi)$ is a continuous function and is uniformly Hölder continuous in $t$; that is, there exist $C>0$ and $\bar{\gamma} \in(0,1)$ such that

$$
\begin{gathered}
\left\|a\left(t_{1}, \xi\right)-a\left(t_{2}, \xi\right)\right\| \leq C\left|t_{1}-t_{2}\right|^{\bar{\gamma}} \\
\xi \in[0,1], 0 \leq t_{1} \leq t_{2} \leq 1 .
\end{gathered}
$$

Define $A(t)$ by

$$
\begin{gathered}
D(A(t))=H^{2}(0,1) \cap H_{0}^{1}(0,1), \\
A(t) u=-a(t, \xi) u^{\prime \prime} .
\end{gathered}
$$

Then $-A(s)$ generates an analytic semigroup $\exp (-t A(s))$ satisfying assumptions (A1)-(A3) ([28]).

For $t \in(0,1], \xi \in[0,1]$, we set

$$
\begin{gathered}
v(t)(\xi)=u(t, \xi), \\
g(v)(\xi)=\sum_{i=0}^{j} \int_{0}^{1} c_{i}(\xi, y) \frac{v\left(t_{i}\right)(y)}{1+v\left(t_{i}\right)(y)} d y, \\
K(t, s)=t-s, \\
f(t, G v(s), v(t))(\xi)=\sin \left(\int_{0}^{t}(t-s) v(s)(\xi) d s\right) \\
+\frac{1}{k \sqrt[k]{t}} \cdot \frac{v(t)(\xi)}{1+v(t)(\xi)},
\end{gathered}
$$

where $1 / \sqrt[k]{t} \in L^{p}\left([0,1], \mathbf{R}^{+}\right)(p>\max \{1 / q, 1 / \bar{\gamma}\})$ and

$$
(G v(t))(\xi)=\int_{0}^{t}(t-s) v(s)(\xi) d s
$$

Now $G^{*}=\sup _{t \in[0,1]} \int_{0}^{t}(t-s) d s=1 / 2<\infty$.

Then (64) can be reformulated as the abstract (4). Moreover,

$$
\begin{array}{r}
\|f(t, G v(t), v(t))(\xi)\| \leq\|G v(t)\|+\frac{1}{k \sqrt[k]{t}}\|v(t)\|, \\
\leq \mu(t)(\|G v(t)\|+\|v(t)\|), \\
\text { a.e. } t \in[0,1],
\end{array}
$$

where $\mu(t)=\max \{1,1 / k \sqrt[k]{t}\}$.

For $v_{1}, v_{2} \in X$, we have

$$
\begin{gathered}
\left\|f\left(t, G v_{1}, v_{1}\right)-f\left(t, G v_{2}, v_{2}\right)\right\| \\
\leq \int_{0}^{t}(t-s)\left\|v_{1}(s)-v_{2}(s)\right\| d s \\
+\frac{1}{k \sqrt[k]{t}}\left\|v_{1}-v_{2}\right\| .
\end{gathered}
$$


Therefore, for any bounded set $D \subset X$, we have

$$
\chi(f(t, G D, D)) \leq \delta(t) \chi(D), \quad \text { for a.e. } t \in[0,1],
$$

where $\delta(t)=\max \{1 / 2,1 / k \sqrt[k]{t}\}$. Now, Theorem 14 implies that the problem (64) has at least a mild solution.

\section{Conclusion}

This paper deals with the existence of mild solution of a class of nonlinear nonautonomous fractional integrodifferential equations with nonlocal conditions in an abstract space. Sufficient conditions for the existence of mild solution are derived with the help of the fixed point theorem for condensing maps. An example is provided to illustrate the obtained result.

\section{Acknowledgments}

The author is grateful to the referees for their valuable suggestions. This work was partly supported by the NSF of China (11201413), the NSF of Yunnan Province (2009ZC054M, 2013FB034), the Educational Commission of Yunnan Province (2012Z010), and the Foundation of Key Program of Yunnan Normal University.

\section{References}

[1] E. Hille and R. S. Phillips, Functional Analysis and Semi-Groups, vol. 31 of American Mathematical Society, American Mathematical Society, Providence, RI, USA, 1957.

[2] A. A. Kilbas, H. M. Srivastava, and J. J. Trujillo, Theory and Applications of Fractional Differential Equations, Elsevier Science, Amsterdam, The Netherlands, 2006.

[3] V. Kiryakova, Generalized Fractional Calculus and Applications, Longman Scientific \& Technical, Harlow, UK, 1994, copublished in the United States with John Wiley \& Sons, New York, NY, USA.

[4] V. Lakshmikantham and A. S. Vatsala, "Basic theory of fractional differential equations," Nonlinear Analysis: Theory, Methods \& Applications, vol. 69, no. 8, pp. 2677-2682, 2008.

[5] K. S. Miller and B. Ross, An Introduction to the Fractional Calculus and Fractional Differential Equations, John Wiley \& Sons, New York, NY, USA, 1993.

[6] S. G. Samko, A. A. Kilbas, and O. I. Marichev, Fractional Integrals and Derivatives, Gordon and Breach Science, New York, NY, USA, 1993.

[7] I. Podlubny, Fractional Differential Equations, Academic Press, San Diego, Calif, USA, 1999.

[8] R. P. Agarwal, V. Lakshmikantham, and J. J. Nieto, "On the concept of solution for fractional differential equations with uncertainty," Nonlinear Analysis: Theory, Methods \& Applications, vol. 72, no. 6, pp. 2859-2862, 2010.

[9] B. Ahmad, J. J. Nieto, A. Alsaedi, and M. El-Shahed, "A study of nonlinear Langevin equation involving two fractional orders in different intervals," Nonlinear Analysis: Real World Applications, vol. 13, no. 2, pp. 599-606, 2012.

[10] K. Balachandran and S. Kiruthika, "Existence of solutions of abstract fractional integrodifferential equations of Sobolev type," Computers \& Mathematics with Applications, vol. 64, no. 10, pp. 3406-3413, 2012.
[11] A. Debbouche, D. Baleanu, and R. P. Agarwal, "Nonlocal nonlinear integrodifferential equations of fractional orders," Boundary Value Problems, vol. 2010, article 78, 2012.

[12] M. M. El-Borai, "The fundamental solutions for fractional evolution equations of parabolic type," Journal of Applied Mathematics and Stochastic Analysis, vol. 2004, no. 3, pp. 197-211, 2004.

[13] G. M. N’Guérékata, “A Cauchy problem for some fractional abstract differential equation with non local conditions," Nonlinear Analysis: Theory, Methods \& Applications, vol. 70, no. 5, pp. 1873-1876, 2009.

[14] L. Hu, Y. Ren, and R. Sakthivel, "Existence and uniqueness of mild solutions for semilinear integro-differential equations of fractional order with nonlocal initial conditions and delays," Semigroup Forum, vol. 79, no. 3, pp. 507-514, 2009.

[15] F. Li, "Existence and uniqueness of mild solution for fractional integrodifferential equations of neutral type with nonlocal conditions," Mathematica Slovaca, vol. 62, no. 5, pp. 921-936, 2012.

[16] E. Hernández, D. O’Regan, and K. Balachandran, “On recent developments in the theory of abstract differential equations with fractional derivatives," Nonlinear Analysis: Theory, Methods \& Applications, vol. 73, no. 10, pp. 3462-3471, 2010.

[17] G. M. Mophou and G. M. N'Guérékata, "Existence of the mild solution for some fractional differential equations with nonlocal conditions," Semigroup Forum, vol. 79, no. 2, pp. 315-322, 2009.

[18] V. E. Tarasov and J. J. Trujillo, "Fractional power-law spatial dispersion in electrodynamics," Annals of Physics, vol. 334, pp. 1-23, 2013.

[19] R. P. Agarwal, B. de Andrade, and G. Siracusa, "On fractional integro-differential equations with state-dependent delay," Computers \& Mathematics with Applications, vol. 62, no. 3, pp. 1143-1149, 2011.

[20] Y. Ren, Y. Qin, and R. Sakthivel, "Existence results for fractional order semilinear integro-differential evolution equations with infinite delay," Integral Equations and Operator Theory, vol. 67, no. 1, pp. 33-49, 2010.

[21] H. Tanabe, Functional Analytic Methods for Partial Differential Equations, Marcel Dekker, New York, NY, USA, 1997.

[22] L. Byszewski, "Theorems about the existence and uniqueness of solutions of a semilinear evolution nonlocal Cauchy problem," Journal of Mathematical Analysis and Applications, vol. 162, no. 2, pp. 494-505, 1991.

[23] J. Liang and T.-J. Xiao, "Semilinear integrodifferential equations with nonlocal initial conditions," Computers \& Mathematics with Applications, vol. 47, no. 6-7, pp. 863-875, 2004.

[24] J. Liang, J. H. Liu, and T.-J. Xiao, "Nonlocal problems for integrodifferential equations," Dynamics of Continuous, Discrete \& Impulsive Systems A, vol. 15, no. 6, pp. 815-824, 2008.

[25] T.-J. Xiao and J. Liang, "Existence of classical solutions to nonautonomous nonlocal parabolic problems," Nonlinear Analysis: Theory, Methods and Applications, vol. 63, no. 5-7, pp. e225e232, 2005.

[26] R. R. Akhmerov, M. I. Kamenskii, A. S. Potapov, A. E. Rodkina, and B. N. Sadovskii, Measures of Noncompactness and Condensing Operators, vol. 55, Birkhäuser, Basel, Switzerland, 1992.

[27] M. Kamenskii, V. Obukhovskii, and P. Zecca, Condensing Multivalued Maps and Semilinear Differential Inclusions in Banach Spaces, vol. 7 of De Gruyter Series in Nonlinear Analysis and Applications, Walter de Gruyter, Berlin, Germany, 2001.

[28] A. Friedman, Partial Differential Equations, Holt, Rinehart and Winston, New York, NY, USA, 1969. 


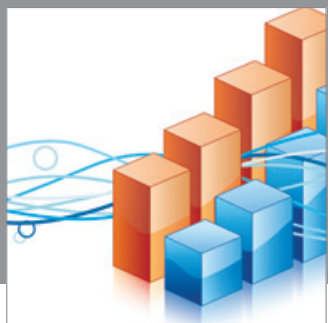

Advances in

Operations Research

mansans

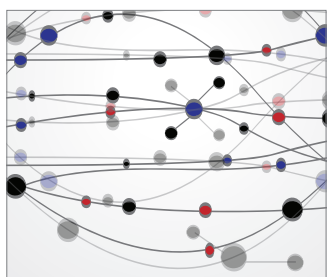

The Scientific World Journal
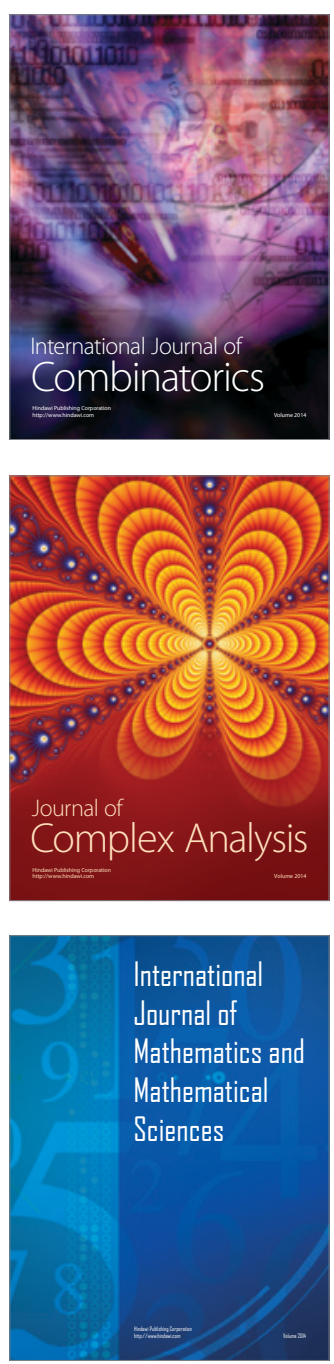
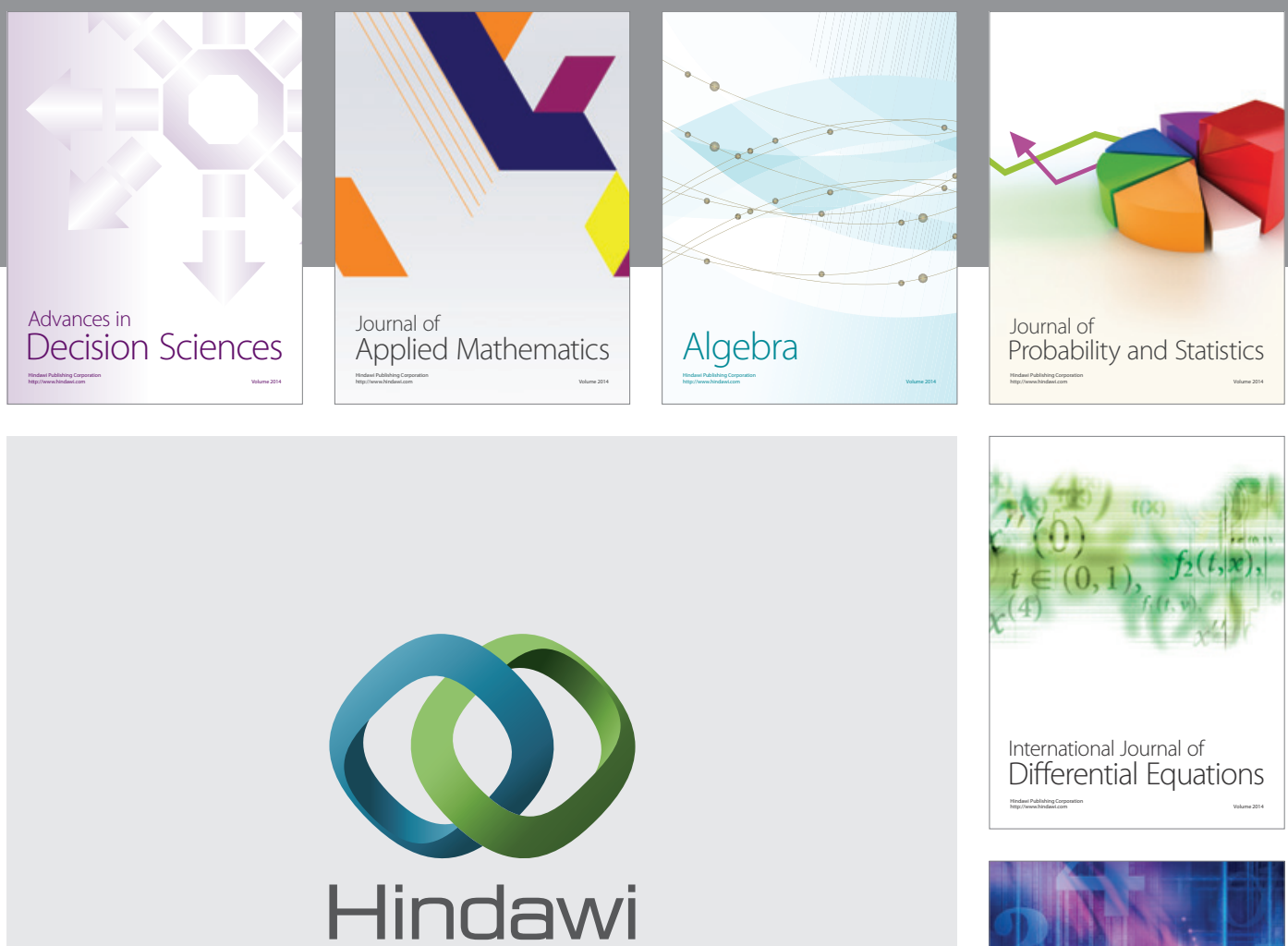

Submit your manuscripts at http://www.hindawi.com
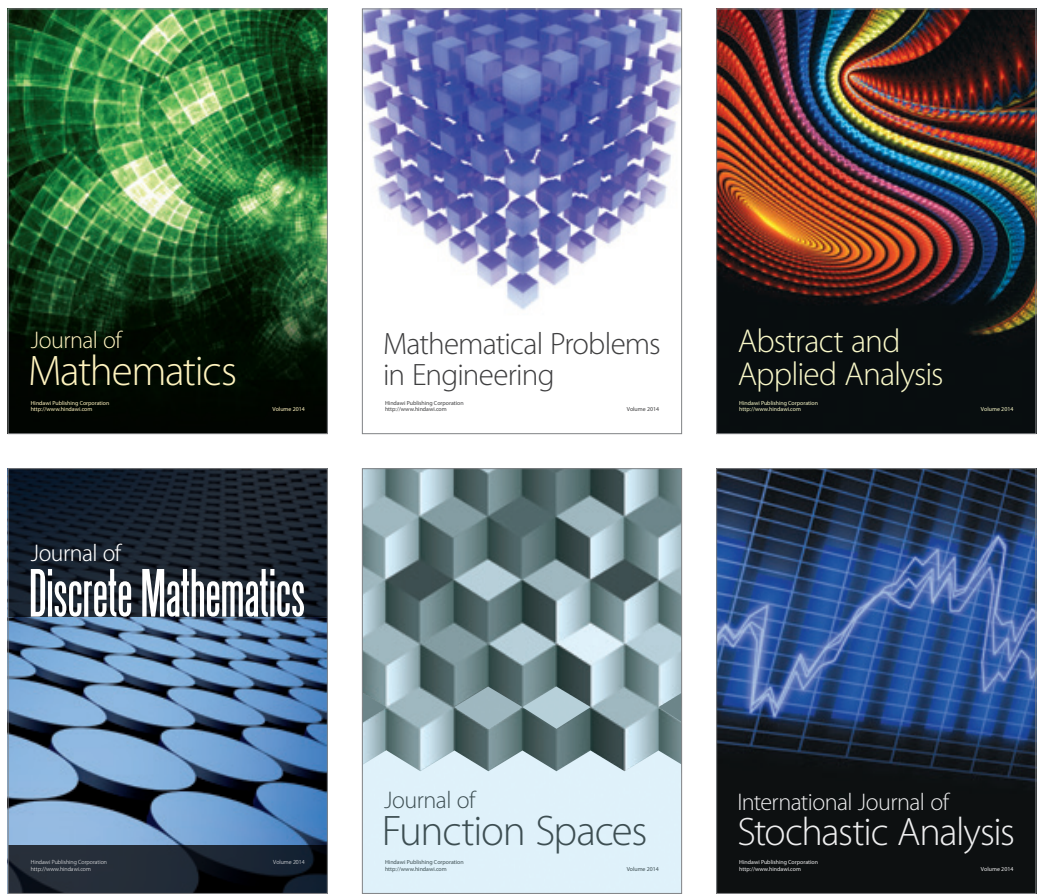

Journal of

Function Spaces

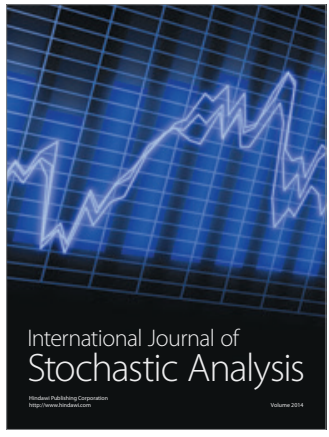

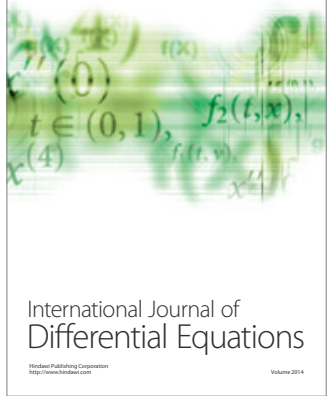
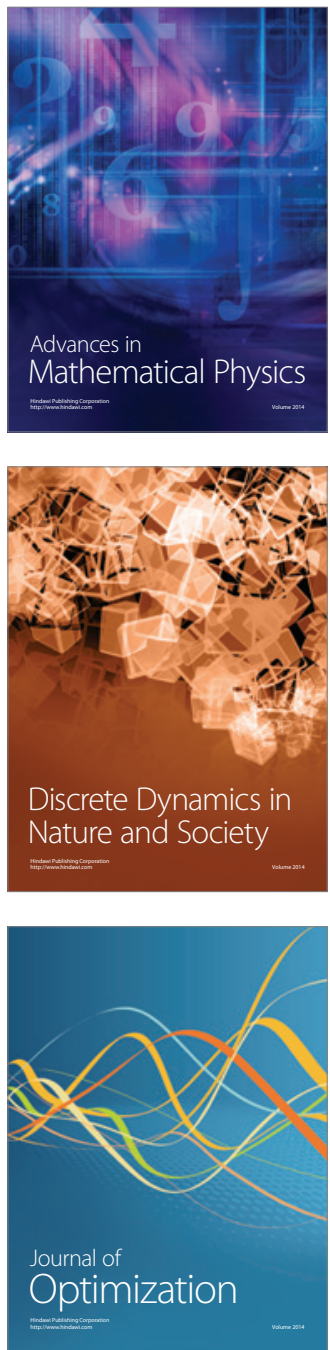